\title{
Structural, optical, and magnetic characterization of monodisperse Fe-doped ZnO nanocrystals
}

\author{
A. Parra-Palomino, ${ }^{1}$ O. Perales-Perez, ${ }^{2, a)}$ R. Singhal, ${ }^{1}$ M. Tomar, ${ }^{1}$ Jinwoo Hwang, ${ }^{3}$ and \\ P. M. Voyles ${ }^{3}$ \\ ${ }_{1}^{1}$ Department of Physics, University of Puerto Rico, Mayagüez, Puerto Rico 00681-9016, USA \\ ${ }^{2}$ Department of Engineering Science and Materials, University of Puerto Rico, Mayagüez, \\ Puerto Rico 00681-9044, USA \\ ${ }^{3}$ Materials Science and Engineering, University of Wisconsin at Madison, Wisconsin 53706-1595, USA
}

(Presented on 6 November 2007; received 11 September 2007; accepted 30 October 2007; published online 14 February 2008)

\begin{abstract}
The results on the synthesis and characterization of highly monodisperse Fe-doped $\mathrm{ZnO}$ nanocrystals are presented. Stable suspensions of these materials were produced in an ethanol solution at room temperature. To promote crystal growth, the suspensions of nanocrystals were aged in contact with their mother liquors. X-ray diffraction characterization of doped systems at various Fe-atomic fractions $x$ confirmed the exclusive formation of the host $\mathrm{ZnO}$ with the wurtzite structure. High resolution transmission electron microscopy analyses of the suspensions revealed the high monodispersity and crystallinity of the $6-8 \mathrm{~nm}$ nanocrystals. Ultraviolet-visible measurements confirmed not only the nanocrystalline nature of the samples but also evidenced the continuous growth of the crystals when aged in their mother liquors. Room-temperature magnetic measurements indicated that the ferromagnetic behavior of doped $\mathrm{ZnO}$ was dependent on composition and crystal size of produced nanocrystals. Room-temperature ferromagnetism was established in the nanocrystals synthesized at $x=0.05$ and 0.08 aged for 24 and $360 \mathrm{~h}$, respectively. The corresponding room-temperature coercivity values were 45 and 78 Oe. (C) 2008 American Institute of Physics. [DOI: 10.1063/1.2834705]
\end{abstract}

\section{INTRODUCTION}

Recent prediction ${ }^{1,2}$ on ferromagnetism in wide band gap semiconductors has opened a broad interest in the emergent field of spintronics. It has been found that the diluted magnetic semiconductors can be formed by replacing the cations of III-V or II-VI semiconductors with transition metal ions. Several researchers reported the experimental evidence of ferromagnetism in transition metal-doped $\mathrm{ZnO}$ thin film and bulk materials produced by different methods. Abid et al. ${ }^{3}$ reported ferromagnetic behavior at $3 \mathrm{~K}$ with coercivity of $137 \mathrm{Oe}$ in $6 \%$ Co-doped $\mathrm{ZnO}$, produced by electrodeposition. Ueda et al. ${ }^{4}$ deposited $\mathrm{Zn}_{1-x} M_{x} \mathrm{O}(x=0.05-0.25 ; M$ $=\mathrm{Co}, \mathrm{Mn}, \mathrm{Cr}, \mathrm{Ni}$ ) films using pulsed laser deposition (PLD) technique. They found that Co-doped $\mathrm{ZnO}$ films exhibited ferromagnetism with a Curie temperature $\left(T_{C}\right)$ above $280 \mathrm{~K}$. Ferromagnetism was also observed for Ni-doped $\mathrm{ZnO}$ nanocrystals. As-synthesized nanocrystals were paramagnetic, but their aggregation gave rise to robust ferromagnetism., Polyakov et al. ${ }^{7}$ observed room-temperature ferromagnetism in Fe- and $\mathrm{Cr}$-implanted bulk $\mathrm{ZnO}$ crystals but after annealing at $700{ }^{\circ} \mathrm{C}$. Optical absorption and microcathodoluminescense measurements suggested the actual incorporation of the transition metal ions. Cho et ll $^{8}$ found ferromagnetic behavior in $(\mathrm{Co}, \mathrm{Fe})$-doped $\mathrm{ZnO}$ film grown by reactive magnetron cosputtering, where the effects of a rapid thermal annealing under vacuum lead to an increase in the magneti-

\footnotetext{
${ }^{a)}$ Electronic mail: ojuan@uprm.edu. Phone: (787)832-4040 x3071. FAX: (787)265-3816.
}

zation and $T_{C}$. Wei et al., ${ }^{9}$ have deposited Fe-doped $\mathrm{ZnO}$ $\left(\mathrm{Zn}_{1-x} \mathrm{Fe}_{x} \mathrm{O}, x=0-0.07\right)$ films onto $\mathrm{LiNbO}_{3}$ substrate by magnetron sputtering and found that Fe substituted the $\mathrm{Zn}$ site in the $2^{+}$charge state when $x \leqslant 0.04$ leading to a strong ferromagnetism $\left(T_{C} \sim 400 \mathrm{~K}\right)$. A further increase of the dopant atomic fraction up to 0.07 caused the precipitation of magnetite $\left(\mathrm{Fe}_{3} \mathrm{O}_{4}\right)$, which was conducive to the increase in the magnetic moment of the material. More recently, Kamakar et al., ${ }^{10}$ produced nanocrystalline $\mathrm{Fe}$-doped $\mathrm{ZnO}$ powders by a chemical pyrophoric reaction method. Magnetization measurements suggested the ferromagnetic to paramagnetic transition temperature to be above $450 \mathrm{~K}$. On the other hand, Mandal et al. ${ }^{11}$ found that the formation of a secondary phase in $\mathrm{Fe}$ and other transition metal ion-doped $\mathrm{ZnO}$ powders was dependent on the level of doping and sample preparation temperature. On this basis, the present work addressed the room-temperature synthesis of highly monodisperse Fe-doped $\mathrm{ZnO}$ nanocrystals in ethanol. The structural, optical, and magnetic characterizations of the synthesized nanocrystals are also presented.

\section{EXPERIMENTAL}

\section{A. Materials}

Zinc acetate, $\mathrm{Fe}(\mathrm{II})$ acetate, $\mathrm{LiOH}$, and anhydrous ethanol were of analytical grade and used without further purification. Suitable amounts of $\mathrm{Zn}$ and $\mathrm{Fe}$ acetate salts were dissolved in ethanol to produce a $0.01 M$ solution. A $\mathrm{LiOH}$ ethanol solution was the precipitant agent. $n$-heptane was used to coagulate nanocrystals from their suspensions. 


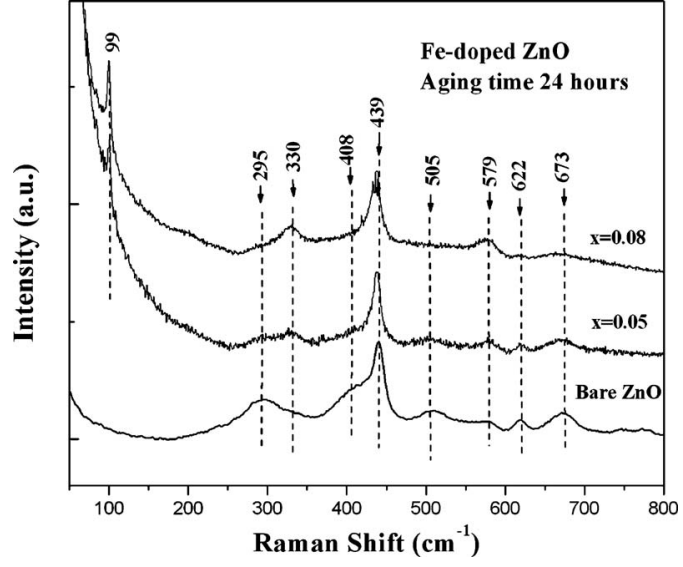

FIG. 1. Raman shift of bare and Fe-doped $\mathrm{ZnO}$ nanocrystals synthesized at different atomic fractions of dopant, " $x . "$

\section{B. Synthesis of nanocrystals}

Doped $\mathrm{ZnO}$ nanocrystals were synthesized in ethanol as reported by Spanhel and Anderson for bare $\mathrm{ZnO}^{12}$ In our case, the syntheses were carried out under ambienttemperature conditions. Metal ions solutions were prepared for a specific dopant atomic fraction, " $x$." The metals and the hydroxide ethanol solutions were mixed under vigorous stirring for $10 \mathrm{~min}$, followed by their aging at room temperature. Nanocrystals were recovered by successive coagulation/ dispersion cycles using $n$-heptane/fresh ethanol and submitted for characterization.

\section{Characterization}

Powders were characterized by x-ray diffraction (XRD) in a Siemens D5000 diffractometer. Raman studies were performed using Jobin-Yvon T64000 spectrophotometer. A Phillips CM200 high resolution transmission electron microscope (HRTEM) was used to study the crystal structure, morphology, and qualitative composition of the nanocrystals. Ultraviolet-visible (UV-Vis) studies were carried out using a Beckman Coulter DU800 spectrophotometer. $M-H$ loops were measured in a Quantum Design MPMS XL-7 supperconducting quantum interference device (SQUID) magnetometer.

\section{RESULTS AND DISCUSSION}

\section{A. XRD diffraction analyses}

All XRD peaks of Fe-doped $\mathrm{ZnO}$ nanocrystals synthesized at different " $x$ " values and different aging times corresponded to $\mathrm{ZnO}$ with the wurtzite structure. The absence of any extra peak in these patterns suggests the actual incorporation of dopant into the host oxide structure. An aging of $24 \mathrm{~h}$ was necessary to complete the development of the oxide structure for " $x$ " values as high as 0.08 . The average crystallite size, estimated by using the Scherrer's equation, was around $4.5 \mathrm{~nm}$. The crystallite size was increased by prolonging the aging period (120 and $360 \mathrm{~h}$ for a concentration of $5 \%$ and $8 \%$ of iron, respectively); the corresponding values varied between 6.5 and $8.3 \mathrm{~nm}$.
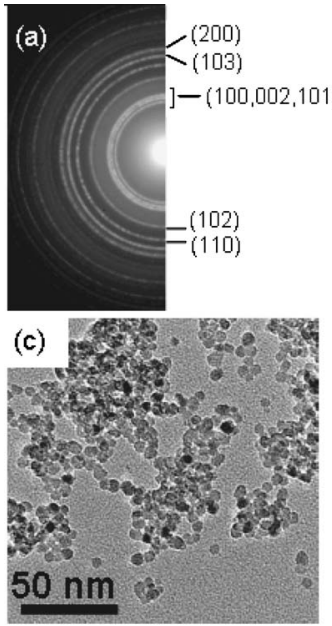

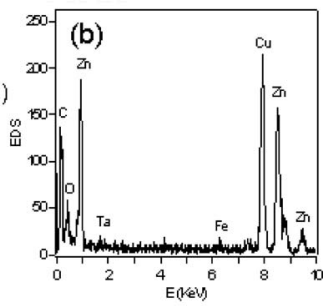

(d)

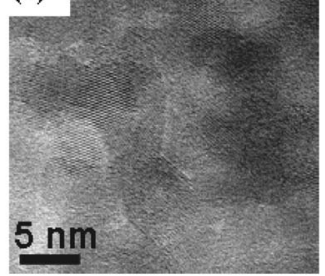

FIG. 2. ED pattern (a), EDS spectrum (b), and HRTEM images [(c) and (d)], of $\mathrm{Fe}-\mathrm{ZnO}$ nanocrystals $(x=0.08)$ aged for 15 days.

\section{B. Raman spectroscopy}

Figure 1 shows the Raman spectra of $\mathrm{Zn}_{1-x} \mathrm{Fe}_{x} \mathrm{O}$ $(x=0.0,0.05,0.08)$ nanocrystals. The phonon modes at 99.5 and $439 \mathrm{~cm}^{-1}$ are assigned to $E_{2}$ (low) and $E_{2}$ (high) vibrational modes, respectively, and are similar as reported for standard $\mathrm{ZnO} .{ }^{13}$ As the concentration of $\mathrm{Fe}$ increases from $5 \%$ to $8 \%$, the $\mathrm{ZnO}$ phonon mode shifted to lower frequencies. It may be due to the decrease in binding energy of $\mathrm{Zn}-\mathrm{O}$ bond as a result of substitution of $\mathrm{Zn}^{2+}$ by $\mathrm{Fe}^{2+}$. The frequencies of other fundamental optical modes in the Fedoped $\mathrm{ZnO}$ were $E_{1} \quad(\mathrm{TO})=408 \mathrm{~cm}^{-1}$ and $A_{1}$ (LO) $=579 \mathrm{~cm}^{-1}$. The intensity of the later mode was enhanced with a rise in the concentration of dopant. These modes were also reported by Wang et al. ${ }^{14}$ The phonon mode at $330 \mathrm{~cm}^{-1}$ is due to a second order spectral feature originated from the zone-boundary phonons of $2-E_{2}(M)$ for $\mathrm{ZnO} .{ }^{15}$ The intensity of the Raman modes located at 295, 505, 622, and $673 \mathrm{~cm}^{-1}$ decreased with a rise in the concentration of the dopant. The reasons behind this trend are currently under investigation and will be a matter of a forthcoming publication.

\section{HRTEM analyses}

Figures 2(a) and 2(b) show, respectively, the electron diffraction (ED) and the energy dispersive x-ray spectroscopy (EDS) patterns of Fe-ZnO nanocrystals $(x=0.08)$ aged for 15 days. All crystallographic planes in the selected-area ED pattern agreed well with those for $\mathrm{ZnO}$, indicating that the Fe-doped $\mathrm{ZnO}$ particles have good crystallinity. No secondary phases were detected. The $\mathrm{Cu}$ and $\mathrm{Ta}$ signals in the EDS are an artifact from either the TEM grid or the sample area of the TEM (pole pieces, sample rod, etc). HRTEM images of the same nanocrystals are shown in Figs. 2(c) and 2(d). The nanocrystals were highly monodisperse, with a diameter averaging $6 \mathrm{~nm}$. Adsorbed acetate species would have contributed to the establishment of a net surface charge onto the nanocrystals preventing their aggregation. ${ }^{5}$ 


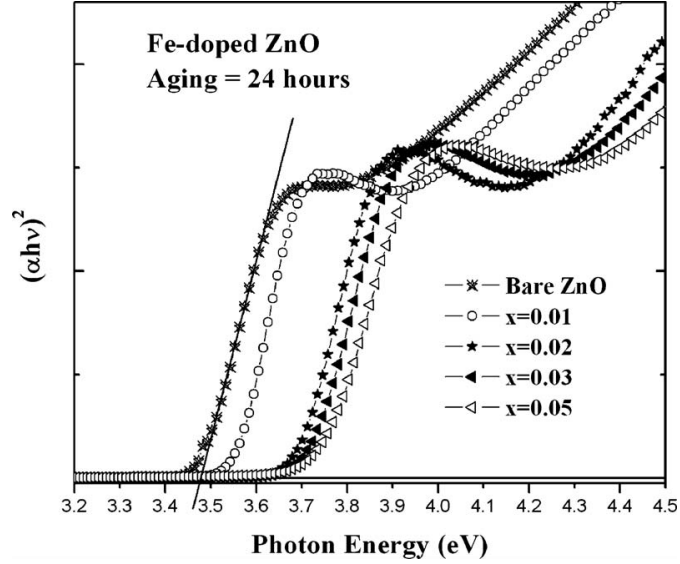

FIG. 3. UV-Vis absorption spectra for nanocrystals synthesized at different atomic fractions of dopant, " $x$," and aged for $24 \mathrm{~h}$.

\section{UV-Vis measurements}

UV-Vis absorption spectra were recorded from stable transparent suspensions of $24 \mathrm{~h}$ aged Fe-doped $\mathrm{ZnO}$ nanocrystals (Fig. 3). The optical band gap was obtained by extrapolating of the linear part until it intersects the $X$ axis. The band edge and the exciton energy shifted to the blue as the concentration of dopant increased from $1 \%$ to $5 \%$. The band gap energy for a concentration of $5 \%$ of $\mathrm{Fe}$ was estimated at $3.74 \mathrm{eV}(335 \mathrm{~nm})$, which is $0.34 \mathrm{eV}$ higher than that for bare $\mathrm{ZnO}(3.4 \mathrm{eV})$ produced at the same aging time. This blueshift can be attributed to the quantum confinement effect in the nanocrystals. ${ }^{16}$ In turn, the rise in the band gap energy values by increasing " $x$ " may suggest the inhibition of crystal growth by the incorporation of dopant species. This trend is in good agreement with our size estimations from XRD measurements of doped $\mathrm{ZnO}$ nanocrystals. In addition to crystal size effect, the variation in the band gap energy can also be caused by the dopant incorporation itself. The dependence of band gap energy with dopant concentration has been observed for $\mathrm{Fe}$ and $\mathrm{Co}$-doped $\mathrm{ZnO}$ (Refs. 17 and 18) films. Although the corresponding results were explained in terms of the distortion of the host lattice and generation of defects, these factors would not play a main role in the determination of the band gap energy because of the negligible variation of the lattice dimensions. ${ }^{19}$ Accordingly, the widening in band gap can more likely be attributed to the $s p$ - $d$ exchange interaction between band electrons and the localized $d$ electrons of Fe substituting Zn ions.

\section{E. SQUID measurements}

Figures 4(a) and 4(b), show the room-temperature (RT) $M-H$ hysteresis loops for Fe-doped $\mathrm{ZnO}$ nanocrystals $(x$ $=0.05,0.08)$ aged for 24 and $360 \mathrm{~h}$. Both samples exhibited a small but evident hysteresis with a coercivity of 45 and $78 \mathrm{Oe}$, and a remnant magnetization of $\sim 2.7 \times 10^{-5}$ and $4.3 \times 10^{-5}$ emu g $^{-1}$, respectively. The formation of magnetite from starting $\mathrm{Fe}(\mathrm{II})$ species could be ruled out under the reducing conditions (provided by ethanol) in our system. Moreover, no second phases were detected in HRTEM analy-
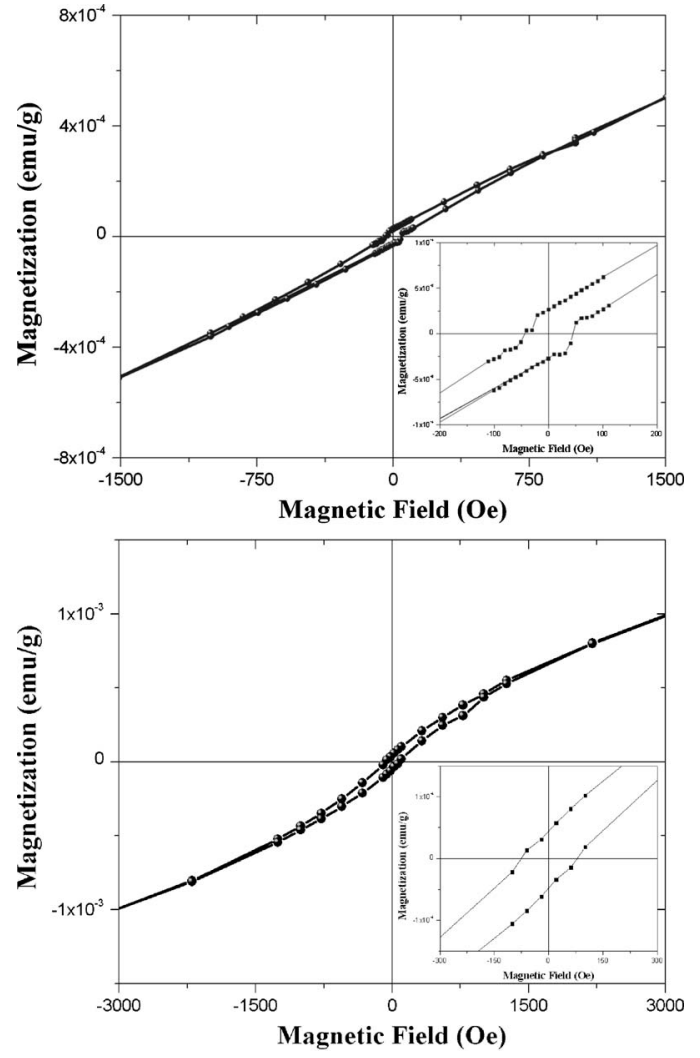

FIG. 4. Room-temperature $M-H$ hysteresis loops for Fe-doped $\mathrm{ZnO}$ nanocrystals, $x=0.05,24 \mathrm{~h}$ aged (a), and $x=0.08,15$ days aged (b).

ses. Our results are in good agreement with those reported for other ferromagnetic $\mathrm{ZnO}$ quantum dots. ${ }^{5,6}$ The generation of lattice defects in $\mathrm{ZnO}$ and/or lattice distortions due to the incorporation of dopant species could be involved with the observed ferromagnetism., 20

\section{ACKNOWLEDGMENTS}

This material is based upon work supported by the National Science Foundation under PREM (Grant Nos. 0351449 -UPRM and 0304479-UW).

${ }^{1}$ K. Sato and H. Katayama-Yoshida, Semicond. Sci. Technol. 17, 367 (2002).

${ }^{2}$ T. Dietl et al., Phys. Rev. B 63, 195205 (2001).

${ }^{3}$ M. Abid et al., J. Electrochem. Soc. 153, D138 (2006).

${ }^{4}$ K. Ueda et al., Appl. Phys. Lett. 79, 988 (2001).

${ }^{5}$ O. Perales-Perez et al., Nanotechnology 18, 315606 (2007).

${ }^{6}$ P. Radovanovic and D. Gamelin, Phys. Rev. Lett. 91, 157202 (2003).

${ }^{7}$ A. Y. Polyakov et al., Mater. Sci. Semicond. Process. 7, 77 (2004).

${ }^{8}$ Y. M. Cho et al., Appl. Phys. Lett. 80, 3358 (2002).

${ }^{9}$ X. X. Wei et al., J. Phys.: Condens. Matter 18, 7471 (2006).

${ }^{10}$ D. Karmakar et al., Phys. Rev. B 75, 144404 (2007).

${ }^{11}$ S. K. Mandal et al., Appl. Phys. Lett. 89, 144105 (2006).

${ }^{12}$ L. Spanhel and M. A. Anderson, J. Am. Chem. Soc. 113, 2826 (1991).

${ }^{13}$ J. Serrano et al., Phys. Rev. B 69, 094306 (2004).

${ }^{14}$ X. B. Wang et al., J. Phys. D 39, 4992 (2006).

${ }^{15}$ J. M. Calleja and M. Cardona, Phys. Rev. B 16, 3753 (1977).

${ }^{16}$ N. S. Norberg et al., J. Am. Chem. Soc. 126, 9387 (2004).

${ }^{17}$ Y. Z. Peng et al., J. Supercond. 18, 97 (2005).

${ }^{18}$ A. J. Chen et al., J. Phys. D 39, 4762 (2006).

${ }^{19}$ Z. W. Zhao et al., Appl. Phys. Lett. 90, 152502 (2007).

${ }^{20}$ C. Song et al., J. Phys.: Condens. Matter 19, 176229 (2007). 